\title{
Cognitive Factors Involved in the Ability to Manipulate a Digital Camera
}

\author{
Keisuke Ishihara, Toshihisa Doi, Sou Yanagimoto, and Toshiki Yamaoka \\ Wakayama University Faculty of Systems Engineering \\ 930, Sakaedani, Wakayama City, Wakayama, 640-8510, Japan \\ \{s125003,s115070,s145064, yamaoka\}@center.wakayama-u.ac.jp
}

\begin{abstract}
The purpose of this study is to understand who user' property affects the ability to manipulate a digital camera. The N-back task, the action control scale, usability test, structural test, functional test, protocol analysis and some questioner are used to understand user' distinction. The relationships among each property and performance were cleared by correlation analysis. As a result, functional models about the camera are most important to use well.
\end{abstract}

Keywords: mental models, working memory, human property.

\section{Introduction}

Recently, many user-interface machines become complex like "black box" by rapid advances in technology and users are becoming extremely troubled with machine operations. Especially, up-to-date machines are becoming more complex. And most operations must be conducted through the operation portion or operation screen. In order to conduct these operations through the operation screen, information is layered and operations are conducted from abstracted information. In this situation, in order to perform user-interface design, a recognizable approach, which unifies the mental models of the user and the designer, are necessary [1]. However, there are great differences between individual's mental models and differ from another in their ability to use interface. When users use a new interface for the first time, it may be easy to use for some user, but some user may not be able to use it. In order to perform easy-to-use user-interface design, it is important to consider how to allow the user to construct a precise mental model. To this end, it is necessary to consider from various viewpoints about the use ability, and to understand their process of constructing a mental model as well as the elements related to cognitive model. So, the purpose of this study is to understand user property affects the ability to manipulate a digital camera. Then, the N-back task, the action control scale, usability test, structural test, functional test, protocol analysis and some questioner are used to understand their distinction. 


\section{Method}

\subsection{Participants}

Participants were 24 students aged 20 to 25 years (men: $\mathrm{M}=22.8, \mathrm{SD}=1.64$; women: $\mathrm{M}=22.8, \mathrm{SD}=1.28$ ). They were either engineering students or university graduates and they had not any previous experience with the target digital camera or similar ones.

\subsection{Experiment Design}

In order to address our research, three types of experiments and 3 type of questionnaire were prepared to understand their distinction of cognitive ability. Each objective describe simply as below:

\section{Experiments:}

(1) The N-Back task was done to measure their working memory.

(2) Usability testing was done to measure their performance.

(3) Two type of mental model's experiment were done to measure their mental models.

Questionnaire:

(4) Questionnaire about operating parts was used to measure their understanding how to use it or how to work each operating parts.

(5) Mental model structural understanding Method (Doi and Yamaoka, 2010) was used to measure their understanding of the equipment.

(6) The action control scale (Kuhl, 1994) was used to measure their action state orientation (human property).

\section{$3 \quad$ Results}

\subsection{The N-Back Task[2]}

The purpose of the first investigation is to measure participants' working memory. The n-back task is a continuous performance task that is commonly used in neuroimaging to stimulate brain activity in test subjects. It was introduced by Wayne Kirchner in 1958. This subject is presented with a sequence of stimuli, and the task consists of indicating when the current stimulus matches the one from $\mathrm{n}$ steps earlier in the sequence. The load factor $\mathrm{n}$ can be adjusted to make the task more or less difficult.

For example, an auditory three-back test could consist of the experimenter reading the following list of letters to the test subject:

T L H C H O C Q L C K L H C Q T R R K C H R 
The subject is supposed to indicate when the letters marked in bold are read, because those correspond to the letters that were read three steps earlier. Sound and Spatial test was done (see figure.1). And, this result shows table.1.

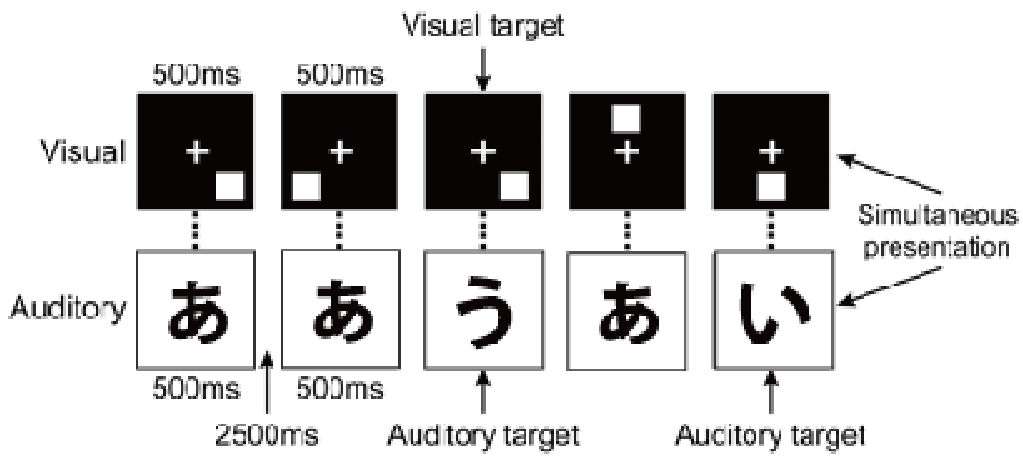

Fig. 1. This is an image of N-Back task. The N-Back task utilizes two main branches in terms of variation, (1) the type of stimulus and (2) the number of turns one must remember 'back.' The type of stimulus commonly falls to audio and spatial. The ' $\mathrm{N}$ ' in N-Back refers to the number of turns back "N" must recall. In our study, this varied from 2-back to as high as around 4-back. A Kolmogorov-Smirnov goodness of fit test was performed to determine whether data were normally distributed.

Table 1. Result of the n-back task

\begin{tabular}{ccrrrrr}
\hline N-back task & $\mathrm{n}$ & \multicolumn{1}{c}{ nean } & $\min$ & $\max$ & \multicolumn{1}{c}{ SD } & p-value \\
\hline visual & 24 & 12.3 & 1 & 24 & 7.26 & $0.5695^{*}$ \\
ouditory & 24 & 12.2 & 1 & 24 & 7.15 & $0.6307^{*}$ \\
\hline
\end{tabular}

\subsection{Usability Testing (Performance and Thinking-Aloud Test)}

This usability testing is a technique used in user-centered interaction design to evaluate a product by testing it on users. This can be seen as an irreplaceable usability practice, since it gives direct input on how real users use the system. This usability testing was focused on measuring manipulate ability. In addition these testing data were changed into numbers to compare other testing data based on some previous studies. Twenty four participants were instructed to perform certain tasks using one digital camera (see figure.2). The tests were conducted in a controlled usability lab by three test administrator. The results of the experiment were registered by the experimental leader using video camera and voice recorder. Participants were given an enough time to finish each task. Instead, these operating were kept track of actual time spent on. 
The experimental tasks were as follows:

- Task A ( "Normal" shooting mode):

Set up the picture quality/size to " $5 M / 4: 3$ ", set up the white balance to "Fluorescent Lamp", change the ISO setting to "100", set up the camera shake correction "ON", and take a picture of the doll on the desk using.

- Task B ("Video move" mode):

Set up the movie size to "QVGA320", set up volume settings "silent mode", take a movie of room for a few seconds, and play the recorded movie.

- Task C ("Continuous" shooting mode):

Set up the picture quality/size to " $1 M / 4: 3$ ", change the white balance and the ISO setting to "Auto", change the image setting to "Black \& White", take a picture of the doll on the desk, and delete 3 photos from the camera.

- Task D ("Scene" shooting mode):

Set up the scene mode to "Zoom Macro", get a macro shot focus on the doll, and take a picture of the doll on the desk using the self-timer (set up 2 seconds).
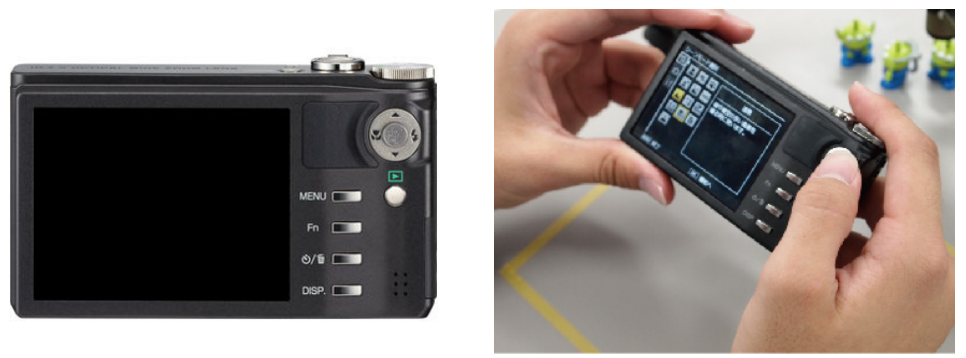

Fig. 2. The digital camera used in this experiment (RICOH CX3)

These four tasks were chosen because they represent common tasks that can be performed on basic modern digital cameras, can be look all menu structure, and can be use a lot of functions of this device during the experiment. These tasks cover a good overview of the various functionalities of digital cameras, ranging from more simple such as take a picture to more complex ones such as using the self-timer settings. If participants repeat some operation or stay in one menu for a long time, they were deemed to unable to do, and they were asked to proceed to the next one.

And then, after experiment participants' performance video data were checked and counted error, and calculated the scores (see table.2). In addition to looking at different types of errors, such as variable versus constant, he focuses on human errors from the standpoint of their intentions, actions and consequences. Many kinds of cognitive idea were observed. We will not take up individual examples in detail. But small problems occur early. For example, some users took no clear judgment. Some users took suspension of operation. Last stage of the problem, they lost where they are (Situation Awareness), and operated the system randomly. Also, some unconsciousness error was observed. 
Table 2. Result of Usability Test

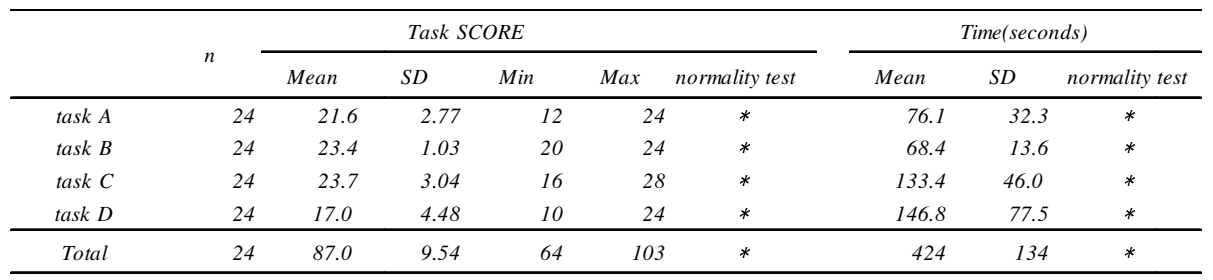

\subsection{Mental Models Test (Structural Model and Functional Model)}

What is mental model? The notion is that a person forms an internal, mental model of themselves and of the things and people with whom they interact. These models provide predictive and explanatory power for understanding the interaction. The term Mental Mode is often used to mean different things, however, which leads to obvious confusion. In particular, the term sometimes refers to the model a user has of the system, sometimes the model the designer has of the system, and sometimes even the model the designer or the system has of the user. In our study, the term refers to the model that the participant has of the digital camera.

And mental model is defined two basic models which are "structural" and "functional" models [3]. Structural models define facts the user has about how the certain camera works. Its basic advantage is that the knowledge of how a device or system works can predict the effect of any possible sequence of actions, meanwhile constructing such a model in mind involves a great deal of effort . On the other hand, functional models, also called task-action mapping models, are procedural knowledge about how to use the camera. The main advantage of functional models is that they can be constructed from existing knowledge about a similar domain or system.
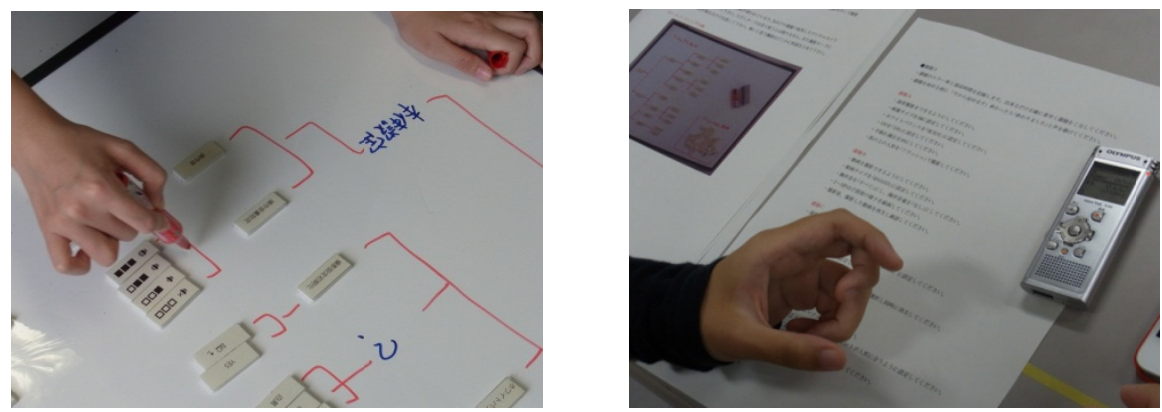

Fig. 3. The left is a Structural model's task and the right is a functional model's task

After usability test, two types of tests which "structural model's task" and "functional model's task" were done to measure their mental models. The experimental tasks were as follows: 
1. Structural model's task: The cards which show illustrations and word one of the menu hierarchies of the digital camera were distributed on the board. The participant must lay all the cards as same as menu hierarchy correctly (see left of figure.3).

2. Functional model's task: The participant told us how to use and how to operate the digital camera which they used in this study. And they explained how to accomplish the each task which they done experiment " 2 " (see right of figure.3).

The result of these experiment show table 3 .

Table 3. Result of mental model experiments

\begin{tabular}{lrrrrrrr}
\hline & $\mathrm{n}$ & \multicolumn{1}{c}{ nean } & $\min$ & $\max$ & \multicolumn{2}{c}{ SD } & p-value \\
\hline S-Task & 24 & 35.8 & 1 & 63 & 16.6 & $0.61 *$ \\
F-Task & 24 & 26.67 & 18 & 36 & 4.6 & $0.86 *$ \\
\hline
\end{tabular}

\subsection{Mental Model Structural Understanding Method}

This is seventeen questionnaires about the mental model which were extracted from investigations of ten product's interfaces by Doi and Yamaoka[4]. Their proposed questionnaire is presented using a five-point Likert item (see table.4). The questionnaire was used to understand participant's mental model structural revels.

Table 4. The sample part of seventeen questionnaires

1. Can understand the terms and words of the menu

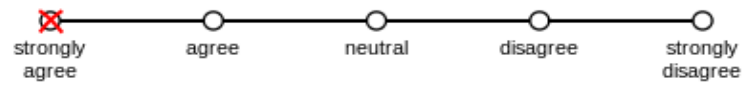

2. Can understand the contents on the screen

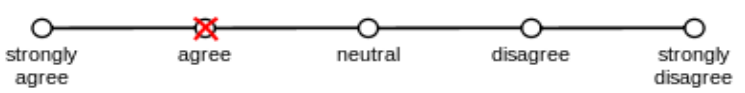

Table 5. Result of Mental model structural understanding Method

\begin{tabular}{ccccccc}
\hline & $\mathrm{n}$ & & nean & $\min$ & $\max$ & \multicolumn{2}{c}{ SD } \\
\hline MM_SUM & & 24 & 61 & 47 & 81 & 9.54 \\
\hline
\end{tabular}

\subsection{Action Control Scale (ACS-90)[5]}

The Action control scale (ACS-90) is designed to measure action-state orientation by Julius Kuhl. The construct is concerned with individual differences in the ability to initiate and maintain intentions / actions. It has also been likened to goal striving as it 
reflects the ability to maintain challenging goals and persist with them despite failures or setbacks. Individuals with a strong action orientation are characterized by enhanced performance efficiency (Kuhl, 1994) and the ability to complete tasks after minor failures or setbacks. While individuals with more of a state orientation tend to have persistent, ruminative thoughts about alternative goals or affective states, which acts to reduce the cognitive resources available for goal-striving, therefore impairing the individual's ability to maintain goal oriented behavior. The action control scale consists of three subscales:

1. Action orientation subsequent to failure vs. preoccupation (AOF)

2. Prospective and decision-related action orientation vs. hesitation (AOD)

3. Action orientation during successful performance of activities intrinsic orientation vs. volatility (AOP)

Participants choose the one of the possible answers (A or B) that is most like them and give an answer for every question on the supplied answer sheet. Each scale consists of 12 items which describe a particular situation (See tabke.1).

Table 6. Sample of ACS-90

\begin{tabular}{ll}
\hline \multicolumn{1}{c}{ A particular situation } & \multicolumn{1}{c}{ answers } \\
\hline 1.When I have lost something valuable and can't find it anywhere: & $\begin{array}{l}\text { A) I have a hard time concentrating on anything else. } \\
\text { B) I don't dwell on it. }\end{array}$ \\
2.When I know I must finish something soon: & A) I have to push myself to get started. \\
& B) I find it easy to get it done and over with. \\
3.When I have learned a new and interesting game: & A) I quickly get tired of it and do something else. \\
& B) I can really get into it for a long time.
\end{tabular}

Scoring \& results: The ACS-90 consists of 36 items, with 12 items for each of the dimensions. The items on the scale depict brief scenarios that occur in everyday life and require selection of one of two options that indicate what the participant would do. Respondents can choose either a ruminative response (scored as 1) or a nonruminative response (scored as 0 ). A total score can range from 0 (no preoccupation) to 12 (extreme rumination). High scores on all 3 dimensions indicate greater actionorientation, while low scores indicate greater state-orientation. Table 2 is a result of our study.

Table 7. Result of ACS-90

\begin{tabular}{cccccr}
\hline $\mathrm{n}$ & mean & SD & $\min$ & \multicolumn{2}{c}{$\max$} \\
\hline 24 & 19.667 & 5.475 & 8 & 30 \\
\hline
\end{tabular}

\section{$4 \quad$ Relationships among All Variables}

All variables were tested for distribution normality using the Kolmogorov-Smirnov test. The Multiple regressions were used to assess the relations among "Manipulation 


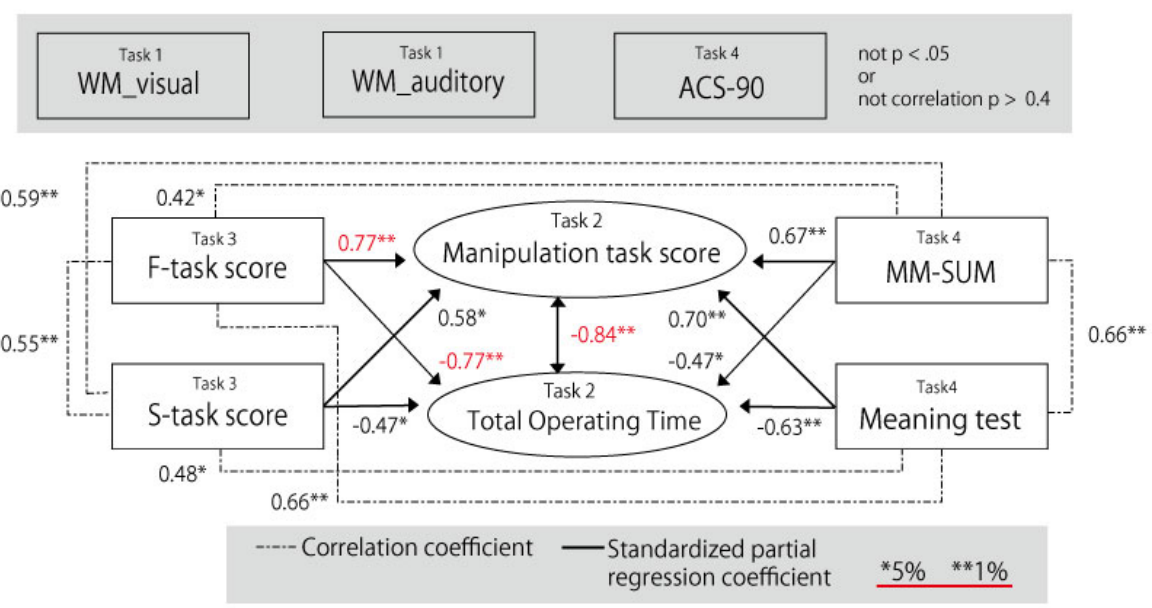

Fig. 4. The relationships among all variables

task score" and other variables. We used "Manipulation task score" as outcome variable, other variables as predictor. Correlation coefficient was obtained using simple regression analysis (Excel software).

The Simple regression analysis revealed the strong correlation between "Manipulation score" and "Total operating time" $(\mathrm{r}=-0.84, \mathrm{P}<.001)$. The correlation between working memory score (n-back task both of visual \& auditory) and manipulation task score was not observed. And significant correlation was not observed between ACS90 score and Manipulation task score $(\mathrm{P}>0.05)$. On the other hand, the strong correlation between "Manipulation score" and "F-task score" was observed ( $\mathrm{r}=-0.77, \mathrm{P}$ $<.001)$. And also, the correlation between "Manipulation score" and "S-task score" was $(\mathrm{r}=-0.58, \mathrm{P}<.005)$, "Manipulation score" and "MM-SUM score" was $(\mathrm{r}=-0.67, \mathrm{P}$ $<.001)$, "Manipulation score" and "Meaning test score was $(\mathrm{r}=-0.63, \mathrm{P}<.001)$ ".

\section{Discussion and Conclusion}

Result of Simple regression analysis and multiple linear regression analysis showed importance of structural models and functional models to good use digital camera. However working memory and Action Control Scale were not important to their performance. It is not clear whether this rule applies to all case. Jenny says structural model is not be needed except when need repairing. The result of our study agrees this opinion. On the other hands, Gentner insist structural importance (structural mapping theory) in terms of analogical process. It is likely that they concentrate consciousness in the use process, when they evaluate the system, even if the process is made after structural mapping. Many type of error were observed. According to Reason's error classification, skill-based error does not relate with thinking. Violation is intentional error. Therefore, knowledge based and rule-based "mistake" has 
relationship with cognitive distance we think. In the future, we would repeat the test using more variety of equipment and interface to increase the reliability and validity of our study.

\section{References}

1. Norman, D.A: Some observations on mental model. In: Genter, D., Stevens, A. (eds.) Mental Models, p. 244. Lawrence Erlbaum Associates Inc., Hillsdale (1983)

2. Kirchner, W.K.: Age differences in short-term retention of rapidly changing information. Journal of Experimental Psychology 55(4), 352-358 (1958)

3. Preece, J., et al.: Structural versus functional models. Human-Computer Interaction, pp. 134-136 (1994)

4. Yamaoka, T., Doi, T., Nishizaki, Y.: A proposal of a user-interface index that corresponds with mental model structural elements. Theoretical Issues in Ergonomics Science 3(2), 135-145 (2012)

5. Kuhl, J.: Volitional aspects of achievement motivation and learned helplessness: Toward a comprehensivetheory of action control. In: Maher, B.A., Maher, W.B. (eds.) Progress in experimental personality research, pp. 99-171. Academic, New York (1984) 